Boeck, Thomas; Zaleski, Stéphane:

Instability of two-phase mixing layers: analysis of exact and approximate base flows from boundary layer theory

URN: $\quad$ urn:nbn:de:gbv:ilm1-2015210146

Published OpenAccess: January 2015

Original published in:

Journal of non-equilibrium thermodynamics : JNETDY. - Berlin [u.a.] : de Gruyter (ISSN 1437-4358). - 30 (2005) 3, S. 215-224.

DOI: $\quad$ 10.1515/JNETDY.2005.017

URL: $\quad$ http://dx.doi.org/10.1515/JNETDY.2005.017

[Visited: 2014-10-15]

„Im Rahmen der hochschulweiten Open-Access-Strategie für die Zweitveröffentlichung identifiziert durch die Universitätsbibliothek Ilmenau."

"Within the academic Open Access Strategy identified for deposition by Ilmenau University Library."

"Dieser Beitrag ist mit Zustimmung des Rechteinhabers aufgrund einer (DFG-geförderten) Allianz- bzw. Nationallizenz frei zugänglich."

"This publication is with permission of the rights owner freely accessible due to an Alliance licence and a national licence (funded by the DFG, German Research Foundation) respectively."

\section{DFG}

Nationallizenzen 


\title{
Instability of two-phase mixing layers: analysis of exact and approximate base flows from boundary layer theory
}

\author{
Thomas Boeck ${ }^{1 *}$ and Stéphane Zaleski ${ }^{2}$ \\ ${ }^{1}$ Fachgebiet Thermo- und Fluiddynamik, TU IImenau, P.O. Box 100565, \\ 98684 Ilmenau, Germany \\ ${ }^{2}$ Laboratoire de Modélisation en Mécanique, Université Pierre et Marie Curie, \\ 4 place Jussieu, case 162, 75252 Paris Cedex 05, France \\ *Corresponding author (thomas.boeck@tu-ilmenau.de) \\ Communicated by E. Michaelides, New Orleans, USA
}

\begin{abstract}
The utility of composite error-function velocity profiles for the modeling of gas-liquid shear layers is examined by comparing temporal stability results from such approximate error-function profiles with results from the exact velocity profiles based on the two-fluid boundary layer equations. The stability analysis is restricted to two-dimensional perturbations. The two-fluid boundary layer equations are solved numerically using a shooting method in each fluid layer. The composite error-function profile is constructed by matching displacement thicknesses with the exact solution. With given fluid properties, the displacement thicknesses depend on the asymptotic velocity ratio of the liquid and gas stream in the laboratory frame. For different sets of fluid properties, the maximum growth rates of the Kelvin-Helmholtz instability show good numerical agreement between the exact and the approximate velocity profiles, especially when the asymptotic velocities of the gas and liquid phase are close.
\end{abstract}

\section{Introduction}

The instability of parallel shear flow is of considerable interest from a fundamental viewpoint and because of its importance for flows in industry and in the environment. The present paper is concerned with the linear instability of 
two-phase shear (mixing) layers, which is important for the atomization of fuels in thermal engines. In atomization experiments and applications, one typically prepares approximately uniform liquid and gas streams with different velocities, which are brought into contact at a nozzle exit or the edge of a splitter plate. Wavy perturbations of the liquid-gas interface grow with increasing downstream distance from the nozzle, and are then drawn out into liquid sheets. Three-dimensional destabilization of these sheets turns them into ligaments, which eventually break up into droplets [1].

Instead of analyzing the spatially growing perturbations, the present work takes the simplest approach to the linear instability. The base flow is assumed parallel and invariant with respect to the streamwise coordinate. Wavy perturbations are assumed as Fourier modes in the streamwise coordinate, and their temporal growth is analyzed. This approach can be adopted as temporal stability results for sinusoidal streamwise perturbations are related to spatially growing perturbations by Gaster's transformation, provided the growth over one oscillation period of the unstable wave is sufficiently small [2]. The consistency of the parallel base flow assumption requires that the crossstream velocity component is insignificant compared with the streamwise velocity, and that the predicted unstable wavelength is substantially smaller than the downstream distance over which the base flow changes significantly. Both requirements may be met when the Reynolds number (based on the downstream distance from the nozzle) is large, in which case the base flow is amenable to boundary layer theory. The base flow should then also approach a self-similar velocity distribution due to the absence of an external lengthscale. Lock [3] has derived ordinary differential equations for this self-similar velocity distribution from the two-fluid boundary layer equations, which evolves from a piecewise constant velocity profile at the origin (nozzle exit). This is shown schematically in Figure 1.

As in the case of one-phase free shear layers, there is no analytical solution for the self-similar velocity distribution. However, an analytical tanh-profile serves as a good approximation of the base flow for stability calculations in the one-phase problem [4]. For this reason, analytical expressions for the base flow have been used in recent works [5-7] instead of Lock's exact solution. The analytical expressions are error functions for the liquid and gas phase. The argument of the error function is scaled with a certain boundary layer thicknesses in each phase, and the zero is located on the interface, i.e., these functions are centered on the interface. Concerning the choice of these analytical expressions, we remark that error functions are approximate solutions to the two-phase boundary layer equations far away from the interface [3] but generally not centered there, and that error functions with time-dependent boundary layer thickness are solutions for parallel flow with 


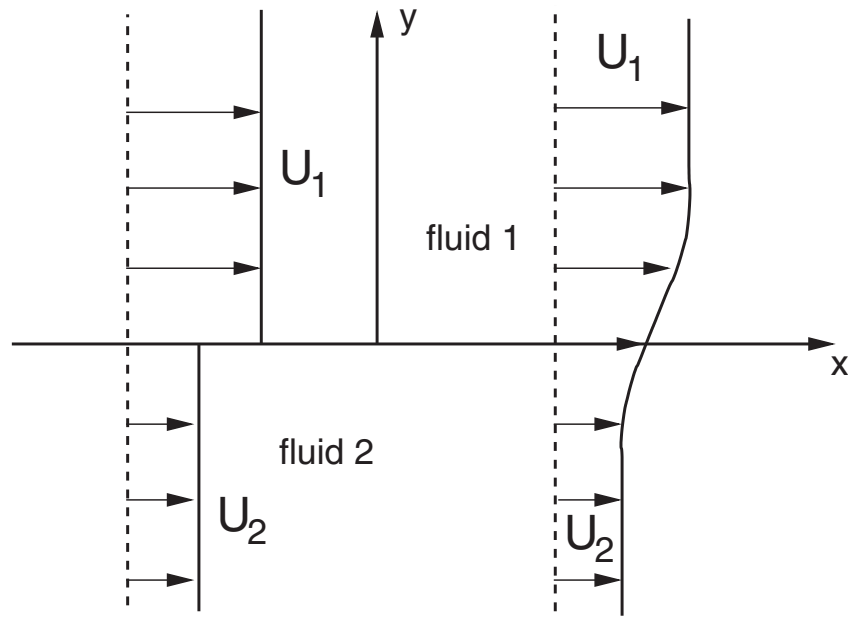

Figure 1 Sketch of the velocity distribution from two-fluid boundary layer analysis.

cross-stream momentum diffusion in the so-called first Stokes problem [8], i.e., the flow next to an impulsively started flat boundary.

The goal of the present paper is to demonstrate the utility of composite errorfunction profiles for the base flow in the temporal stability analysis of twophase shear layers. For this purpose, we shall compare stability results for composite error-function profiles with results for the profiles from Lock's theory. We note that the publications [5-7] were all concerned with the linear stability of the composite error-function velocity profiles, but they did not study the profiles from Lock's theory. Yecko et al. [5] started the investigation by analyzing the instability of two-dimensional perturbations, and Boeck and Zaleski [7] built on this by examining the effects of viscosity through viscous and inviscid stability computations. Yecko and Zaleski [6] go beyond traditional stability analysis in terms of eigenvalues by analyzing optimal temporal growth of three-dimensional perturbations [9].

The present paper is concerned with a traditional stability analysis of twodimensional perturbations. In the next section, we formulate the boundary layer equations and describe our numerical method for solving them. We then compute the displacement thicknesses of the two boundary layers next to the interface in each fluid for the exact velocity profile, and define the corresponding composite error-function profiles with identical displacement thicknesses. We also consider the variation of the displacement thicknesses with the fluid properties and the asymptotic velocities far away from the interface. In the final section, the temporal stability problem is formulated and stability results for the exact profiles and corresponding approximate profiles are compared. 


\section{Two-fluid boundary layer equations}

The boundary layer equations for the two-fluid problem are [3]

$$
u \frac{\partial u}{\partial x}+v \frac{\partial u}{\partial y}=v_{1} \frac{\partial^{2} u}{\partial y^{2}} \quad(y>0), \quad u \frac{\partial u}{\partial x}+v \frac{\partial u}{\partial y}=v_{2} \frac{\partial^{2} u}{\partial y^{2}} \quad(y<0)
$$

where the two fluids are labeled 1 and 2, and $v$ denotes the kinematic viscosity. The two velocity components $u$ and $v$ satisfy the two-dimensional continuity equation

$$
\partial u / \partial x+\partial v / \partial y=0
$$

and can be represented by a streamfunction $\psi$ with

$$
u=\frac{\partial \psi}{\partial y}, \quad v=-\frac{\partial \psi}{\partial x}
$$

in each fluid. For the similarity solution, one introduces the nondimensional similarity variables

$$
\eta_{1}=y \sqrt{\frac{U_{1}}{v_{1} x}} \quad(y>0), \quad \eta_{2}=y \sqrt{\frac{U_{1}}{v_{2} x}} \quad(y<0),
$$

which are then used in the ansatz

$$
\psi=\sqrt{x U_{1} v_{1}} f_{1}\left(\eta_{1}\right) \quad(y>0), \quad \psi=\sqrt{x U_{1} v_{2}} f_{2}\left(\eta_{2}\right) \quad(y<0)
$$

for the streamfunction in each layer. The velocities $U_{1}$ and $U_{2}$ are the asymptotic values of the velocities of the flows far away from their interface (cf. Figure 1). Using the streamfunction ansatz in the boundary layer equations (1), one obtains the ordinary differential equations

$$
2 \frac{\mathrm{d}^{3} f_{1}}{\mathrm{~d} \eta_{1}^{3}}+f_{1} \frac{\mathrm{d}^{2} f_{1}}{\mathrm{~d} \eta_{1}^{2}}=0 \quad\left(\eta_{1}>0\right), \quad 2 \frac{\mathrm{d}^{3} f_{2}}{\mathrm{~d} \eta_{2}^{3}}+f_{2} \frac{\mathrm{d}^{2} f_{2}}{\mathrm{~d} \eta_{2}^{2}}=0 \quad\left(\eta_{2}<0\right) .
$$

Each of these nonlinear, third-order equations requires three boundary conditions. One condition is that the dividing streamline $\psi=0$ coincides with the interface $y=0$, i.e., $f_{1}(0)=f_{2}(0)=0$. Two other conditions on the interface are the continuity of the tangential velocity $u$ and the shear stress, which take the form

$$
f_{1}^{\prime}(0)=f_{2}^{\prime}(0), \quad \sqrt{\rho_{1} \mu_{1}} f_{1}^{\prime \prime}(0)=\sqrt{\rho_{2} \mu_{2}} f_{2}^{\prime \prime}(0),
$$


where $\rho$ and $\mu$ denote the density and the dynamic viscosity. The final two conditions prescribe the limiting values of the streamwise velocity component $u$ far away from the interface, i.e.,

$$
f_{1}^{\prime}(\infty)=1, \quad f_{2}^{\prime}(-\infty)=\lambda=\frac{U_{2}}{U_{1}} .
$$

We solve the equations for $f_{1}$ and $f_{2}$ by integrating them numerically with initial values at the interface $\eta_{1}=\eta_{2}=0$. Besides the given values $f_{1}(0)=$ $f_{2}(0)=0$, we prescribe values for $f_{1}^{\prime \prime}(0), f_{2}^{\prime \prime}(0)$, and $f_{1}^{\prime}(0)=f_{2}^{\prime}(0)$. For fixed $f_{1}^{\prime}(0)=f_{2}^{\prime}(0)$, we adjust $f_{1}^{\prime \prime}(0), f_{2}^{\prime \prime}(0)$ by fixed point iteration such that the asymptotic values for $f^{\prime}$ are met far away from the interface in each fluid. This way, all conditions are satisfied except for the shear stress continuity. The zero in the shear stress difference $\sqrt{\rho_{1} \mu_{1}} f_{1}^{\prime \prime}(0)-\sqrt{\rho_{2} \mu_{2}} f_{2}^{\prime \prime}(0)$ is found through bisection with respect to the remaining independent variable $f_{1}^{\prime}(0)$, which represents the dimensionless interface velocity. For the numerical integration we use a straightforward Adams-Bashforth method.

\section{Approximate velocity profiles}

The streamwise velocity from the boundary layer equations is given by $u=U_{1} f_{1}^{\prime}\left(\eta_{1}\right)$ for $y>0$ and $u=U_{1} f_{2}^{\prime}\left(\eta_{2}\right)$ for $y<0$. For the stability problem we consider the profile $U(y)$ of the streamwise velocity at a fixed position $x>0$. This profile will be approximated by error functions centered at the interface, namely by

$$
\begin{aligned}
& U_{a}(y)=\left(U_{1}-U_{0}\right) \operatorname{erf}\left(y / \delta_{1}\right)+U_{0} \quad(y>0), \\
& U_{a}(y)=\left(U_{0}-U_{2}\right) \operatorname{erf}\left(y / \delta_{2}\right)+U_{0} \quad(y<0),
\end{aligned}
$$

where $U_{0}$ denotes the velocity at the interface. The boundary layer thicknesses $\delta_{1}$ and $\delta_{2}$ are chosen such that the approximate profile $U_{a}(y)$ has the same displacement thickness in each fluid as the exact solution $U(y)$. The displacement thicknesses in the fluids 1 and 2 are defined by

$$
\frac{1}{U_{1}-U_{0}} \int_{0}^{\infty}\left(U_{1}-U(y)\right) \mathrm{d} y, \quad \frac{1}{U_{0}-U_{2}} \int_{-\infty}^{0}\left(U(y)-U_{2}\right) \mathrm{d} y .
$$

Ideally, the approximate solution $U_{a}$ should also satisfy the shear stress continuity condition on the interface, i.e., $\mu_{1}\left(U_{1}-U_{0}\right) / \delta_{1}=\mu_{2}\left(U_{0}-U_{2}\right) / \delta_{2}$. We can judge the quality of the approximation from the shear stress ratio $q$ at the interface given by 


$$
q=\frac{\mu_{1}}{\mu_{2}} \frac{\delta_{2}}{\delta_{1}} \frac{U_{1}-U_{0}}{U_{0}-U_{2}}=\frac{m s}{n}, \quad m=\frac{\mu_{1}}{\mu_{2}}, \quad n=\frac{\delta_{1}}{\delta_{2}}, \quad s=\frac{U_{1}-U_{0}}{U_{0}-U_{2}}
$$

For the definition of $q$ we have introduced the dimensionless ratios $m, n$, and $s$ for the dynamic viscosities, boundary layer thicknesses, and asymptotic velocities (in the co-moving reference frame with velocity $U_{0}$ ). We also introduce $r$ as the density ratio $r=\rho_{1} / \rho_{2}$.

Figure 2 shows the dependency of $n$ and $q$ on the velocity ratio $\lambda$ in the laboratory frame for two sets of parameters $r$ and $m$. Shear stress balance is realized for $q=1$. The largest departure of $q$ from unity occurs for $\lambda \rightarrow 0$. The values of $n$ are of order unity even though $m$ and $r$ are fairly small compared

(a)

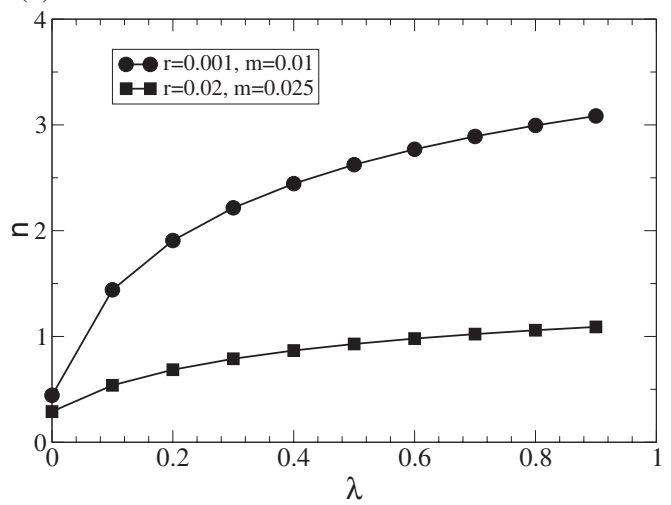

(b)

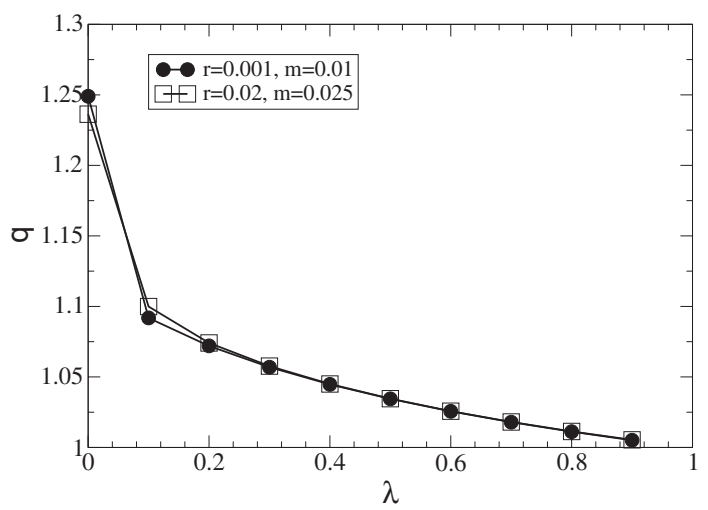

Figure 2 Ratio $n$ of boundary layer thicknesses (a) and ratio $q$ of gas and liquid shear stresses on the interface (b) as functions of the asymptotic velocity ratio $\lambda$ for parameters corresponding approximately to air/water $(r=0.001, m=0.01)$ and hydrogen/liquid oxygen $(r=0.02$, $m=0.025)$. 
with unity. Except for $\lambda=0$, differences between the numerical solution $U(y)$ and the approximation $U_{a}(y)$ are hardly visible when plotted. For this reason, we do not include plots of $U(y)$.

\section{Temporal stability problem}

The temporal stability is governed by the Orr-Sommerfeld equations, which take the nondimensional form [5]

$$
\begin{aligned}
& (U-c)\left(D^{2}-\alpha^{2}\right) \phi_{1}-D^{2} U \phi_{1}=\frac{1}{i \alpha R e}\left(D^{2}-\alpha^{2}\right)^{2} \phi_{1} \quad(y>0), \\
& (U-c)\left(D^{2}-\alpha^{2}\right) \phi_{2}-D^{2} U \phi_{2}=\frac{r}{m} \frac{1}{i \alpha \operatorname{Re}}\left(D^{2}-\alpha^{2}\right)^{2} \phi_{2} \quad(y<0) .
\end{aligned}
$$

In these equations, $U$ represents either the exact velocity profile $U(y)$ or the approximate velocity profile $U_{a}(y)$; and $\phi$ denotes the $y$-dependence of the streamfunction perturbations

$$
\begin{aligned}
& \Psi_{1}(x, y, t)=\exp (i \alpha(x-c t)) \phi_{1}(y) \quad(y>0), \\
& \Psi_{2}(x, y, t)=\exp (i \alpha(x-c t)) \phi_{2}(y) \quad(y<0)
\end{aligned}
$$

to the base flow. The symbol $D$ denotes the derivative with respect to the coordinate $y, \alpha$ denotes the wavenumber, and $c$ the complex phase velocity.

The boundary conditions at the interface $y=0$ are derived in [10]. They comprise the continuity of the normal and tangential velocity, i.e.,

$$
\phi_{1}(0)=\phi_{2}(0), \quad D \phi_{1}+D U\left(0^{+}\right) \frac{\phi_{1}(0)}{c}=D \phi_{2}(0)+D U\left(0^{-}\right) \frac{\phi_{2}(0)}{c},
$$

and the continuity of normal and tangential stress, i.e.,

$$
\begin{aligned}
&-\frac{\alpha^{2}}{c W e} \phi_{1}=-\frac{1}{r}\left(c D \phi_{2}+\phi_{2} D U\right)-\frac{1}{m} \frac{1}{i \alpha R e}\left(D^{3}-3 \alpha^{2} D\right) \phi_{2} \\
&+c D \phi_{1}+\phi_{1} D U+\frac{1}{i \alpha R e}\left(D^{3}-3 \alpha^{2} D\right) \phi_{1}, \\
& m\left(D^{2}+\alpha^{2}+\frac{1}{c} D^{2} U\right) \phi_{1}=\left(D^{2}+\alpha^{2}+\frac{1}{c} D^{2} U\right) \phi_{2} .
\end{aligned}
$$


Notice that these dimensionless equations and boundary conditions are based on the length and velocity scales of fluid 1, i.e., on $\delta_{1}$ and $U_{1}-U_{0}$. The Reynolds number $R e$ and the Weber number $W e$ are defined as

$$
R e=\frac{\rho_{1}\left(U_{1}-U_{0}\right) \delta_{1}}{\mu_{1}}, \quad W e=\frac{\rho_{1}\left(U_{1}-U_{0}\right)^{2} \delta_{1}}{\sigma},
$$

where $\sigma$ denotes the interfacial tension. The Orr-Sommerfeld equations represent a linear eigenvalue problem for $c$ with given nondimensional velocity profile $U$ and given values for $R e, W e$, the various ratios of material properties, and the wave number $\alpha$. It is solved numerically using a Chebyshev collocation method [11]: One assumes additional rigid walls at $y=-L_{2}, y=L_{1}$ and expands the eigenfunctions $\phi_{1}(y)$ and $\phi_{2}(y)$ in Chebyshev polynomials on the intervals $\left[0, L_{1}\right]$ and $\left[-L_{2}, 0\right]$. The algebraic eigenvalue problem is obtained by substitution of the expansions into the Eqs. (12) and (13) for $\phi_{1}$ and $\phi_{2}$ and evaluation of (12) and (13) at collocation points chosen to ensure spectral accuracy. For the solution of the resulting general algebraic eigenvalue problem, we use the NAG library function F02GJF. Both $L_{1}$ and $L_{2}$ need to be sufficiently large to ensure that the results are independent of the choice for these parameters.

In the following, the imaginary part of the complex eigenvalue $c$ will be denoted by $c_{i}$. The growth rates $\alpha c_{i}$ of the unstable modes are shown in Figure 3 as function of $\alpha$ for the parameters $m$ and $r$ used in Figure 2. The values for $m$ and $r$ correspond approximately to the fluid combinations air/water and hydrogen/liquid oxygen, which are used in laboratory atomization experiments and in cryogenic rocket engines, respectively. The chosen Reynolds and Weber numbers are also in the typical range for these applications. The diagrams compare results for the exact and approximate velocity profiles $U$ and $U_{a}$ for $\lambda=0$ and $\lambda=0.9$. We see from Figure 3(a) and 3(c) that there is a noticeable difference in the growth rates for $\lambda=0$ between the exact and approximate profiles, whereas there is no visible difference for $\lambda=0.9$ in Figure $3(\mathrm{~b})$ and $3(\mathrm{~d})$. The improving agreement between the growth rates from exact and approximate profiles with growing $\lambda$ is in line with the observation $q(\lambda) \rightarrow 1$ as $\lambda \rightarrow 1$ shown in Figure 2(b). We can conclude that the error-function approximation works very well in this limit. Another interesting observation is that the thickness of the boundary layer in fluid 2 does not seem to have much effect on the results. This is demonstrated by Figures $3(\mathrm{~b})$ and $3(\mathrm{~d})$, where the curves for $\lambda=0$ and $\lambda=0.9$ are fairly close although the thickness ratio $n$ differs by a factor of three or more. The reasons for this behavior are discussed in [7]. 
(a)

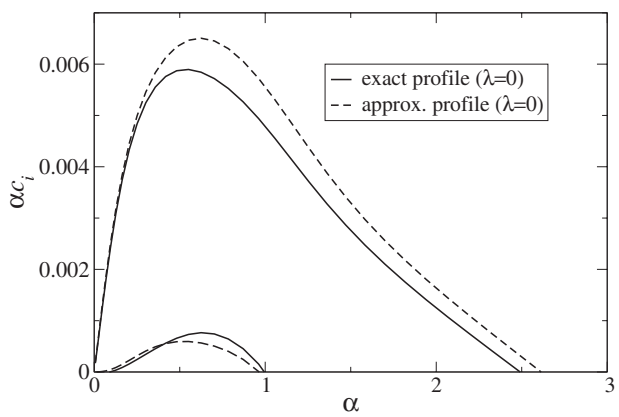

(c)

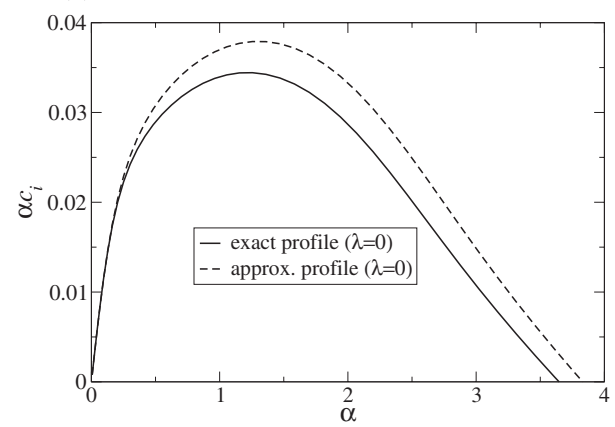

(b)

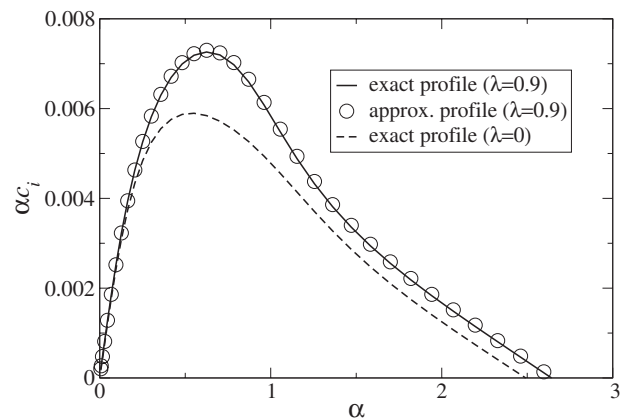

(d)

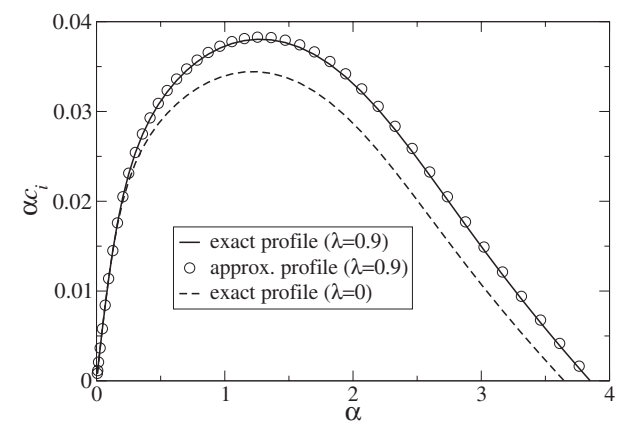

Figure 3 Unstable modes computed with exact and approximate velocity profiles for $r=0.001, m=0.01, R e=500, W e=10(\mathrm{a}, \mathrm{b})$ and for $r=0.02, m=0.025, R e=1000$, $W e=100(\mathrm{c}, \mathrm{d})$. We display all the unstable modes for $\lambda=0$ in (a) and (c), of which there are two in (a) and one in (c). For $\lambda=0.9$, we show the single unstable mode in (b) and (d). The most unstable mode for $\lambda=0$ is shown as a dashed line in (b) and (d) for comparison.

\section{Acknowledgment}

The authors thank Philip Yecko for sharing his stability codes. T.B. is grateful for the hospitality of the research group "Magnetofluiddynamics" at the University of Ilmenau, and for financial support through an Emmy Noether fellowship of the Deutsche Forschungsgemeinschaft. S.Z. acknowledges support from CNES and SNECMA through the GDR moteurs fusées.

\section{References}

[1] Lasheras, J.C., Hopfinger, E.J., Liquid jet instability in a coaxial gas stream, Annu. Rev. Fluid Mech., 32 (2000), 275-308.

[2] Gaster, M., A note on the relation between temporally-increasing and spatiallyincreasing disturbances in hydrodynamic stability, J. Fluid Mech., 14 (1962), $222-224$. 
[3] Lock, R.C., The velocity distribution in the laminar boundary layer between parallel streams, Q. J. Mech. Appl. Math., 4 (1951), 42-63.

[4] Monkewitz, P.A., Huerre, P., Influence of the velocity ratio on the spatial instability of mixing layers, Phys. Fluids, 25 (1982), 1137-1143.

[5] Yecko, P., Zaleski, S., Fullana, J.-M., Viscous modes in two-phases mixing layers, Phys. Fluids, 14 (2002), 4115-4122.

[6] Yecko, P., Zaleski, S., Transient growth in two-phases mixing layers, J. Fluid Mech., 528 (2005), 43-52.

[7] Boeck, T., Zaleski, S., Viscous versus inviscid instability of two-phase mixing layers with continuous velocity profile, Phys. Fluids, 17 (2005), 032106.

[8] Schlichting, H., Boundary Layer Theory, pp. 90-91, McGraw-Hill, New York, 1987.

[9] Trefethen, L.N., Trefethen, A.E., Reddy, S.C., Driscoll, T.A., Hydrodynamic stability without eigenvalues, Science, 261 (1993), 578-584.

[10] Yih, C.S., Instability due to viscosity stratification, J. Fluid Mech., 67 (1967), 337-352.

[11] Schmid, P.J., Henningson, D.S., Stability and Transition in Shear Flows, Appendix A, Springer-Verlag, New York, 2001.

Paper received: 2005-01-14

Paper accepted: 2005-04-05 